\title{
Prostate-MRI: experience of the observer and technical conditions influence the cancer detection rate
}

\author{
S Roedel', S Blaut, E Duerig, M Burke, R Paulick, G Haroske, F Steinbach, T Kittner \\ From International Cancer Imaging Society (ICIS) 14th Annual Teaching Course \\ Heidelberg, Germany. 9-11 October 2014
}

\begin{abstract}
Aim
The prostate cancer (PCa) detection rate of MR-guided biopsy (MRGB) increased in our hospital from $36.6 \%$ in 2012 to $69 \%$ in 2013 . The study analysed the values of the mpMRI-characteristics of identifiable lesions retrospectively to show the influence of the increasing experience of the observer and modified technical conditions.
\end{abstract}

\section{Methods}

56 patients (pat.) with mostly at least one prior negative TRUS-guided biopsy and persistent suspicion of PCa with at least one mpMRI-defined identifiable cancer suspicious lesion were included in this study between 2012 and 2013. MpMRI: $1.5 \mathrm{~T} / \mathrm{e}$-coil/T2WI/DWI, b-values 2012: 0-1500, 2013: 100-1500/DCE-MRI]. MRGB: inbore. Characteristics of lesions (ADC, ESUR PIRADS) were statistically correlated with core needle biopsy results (ROC). A p value of $p<0.05$ was considered as statistically significant.

\section{Results}

2012/2013: detection rate of all suspicious lesions 33\%/ $58 \%$; in peripheral zone $45 \% / 50 \%$; in transitional zone $14 \% / 67 \%$. The ROC curve area difference was statistically significant for 2012/2013 for ADC 0.65/0.83 ( $\mathrm{P}=0.008)$. The cut-off values [cut-off (sensitivity; specificity)]: 2012/ 2013: ADC 836 (0.58;0.58) / 651 (0.72;0.71); 2013: PIRADS DWI $3.5(0.57 ; 1.0)$, PIRADS DCE $3.5(0.63 ; 0.69)$, PIRADS T2 $3.5(0.71 ; 0.86)$.

\section{Conclusion}

Modified DWI as to exclude microcapillary perfusion effects leads to lower cut-off value and higher diagnostic value of the $A D C$. The increasing experience of the observer enhances the evaluation of the transitional zone. The combination of the modified technical conditions and increasing experience of the observer leads to higher sensitivity and specificity of the overall mpMRI prostate evaluation and $(\mathrm{PCa})$ detection rate.

Published: 9 October 2014

doi:10.1186/1470-7330-14-S1-S2

Cite this article as: Roedel et al:: Prostate-MRI: experience of the observer and technical conditions influence the cancer detection rate. Cancer Imaging 2014 14(Suppl 1):S2.

* Correspondence: drstroedel@aol.com Krankenhaus Dresden-Friedrichstadt 